Check for updates

Cite this: RSC Adv., 2018, 8, 31943

Received 6th August 2018

Accepted 3rd September 2018

DOI: $10.1039 / \mathrm{c} 8 \mathrm{ra06595k}$

rsc.li/rsc-advances

\section{Functionalized carboxylate deposition of triphenylamine-based organic dyes for efficient dye-sensitized solar cells}

\author{
Md Ataul Mamun, (DD ${ }^{\mathrm{b}}$ Qiquan Qiao (iD) ${ }^{\mathrm{b}}$ and Brian A. Logue*a
}

The standard dip-coating dye-loading technique for dye-sensitized solar cells (DSSCs) remains essentially unchanged since modern DSSCs were introduced in 1991. This technique constitutes up to $80 \%$ of the DSSC fabrication time. Dip-coating of DSSC dyes not only costs time, but also generates a large amount of dye waste, necessitates use of organic solvents, requires sensitization under dark conditions, and often results in inefficient sensitization. Functionalized Carboxylate Deposition (FCD) was introduced as an alternative dye deposition technique, requiring only $2 \%$ of the fabrication time, eliminating the need for solvents, and significantly reducing dye waste. In this study, FCD was used to deposit two relatively large triphenylamine-based organic dyes (L1 and L2). These dyes were sublimated and deposited in $<20$ minutes via a customized FCD instrument using a vacuum of $\sim 0.1 \mathrm{mTorr}$ and temperatures $\leq 280{ }^{\circ} \mathrm{C}$. FCD-based DSSCs showed better efficiency (i.e., 5.03\% and 5.46\% for L1 and L2 dyes, respectively) compared to dip-coating (i.e., $4.36 \%$ and $5.35 \%$ for $L 1$ and $L 2$, respectively) in a fraction of the deposition time. With multiple advantages over dip-coating, FCD was shown to be a viable alternative for future ultra-low cost DSSC production.

\section{Introduction}

Dye sensitized solar cells (DSSCs) are produced from low-cost materials, their fabrication does not require clean room/glovebox conditions, and DSSCs have produced power conversion efficiencies (PCEs) of more than 11\%. ${ }^{1-3}$ Although the advantages of DSSCs are promising, their fabrication requires some high-cost materials, long fabrication times, and the use of organic solvents. For example, organometallic dyes are commonly used to produce high efficiency DSSCs. ${ }^{4,5}$ These dyes typically require complex synthetic and purification processes. Although progress has been made towards higher-efficiency DSSCs, the state-of-the-art dye loading technique, dip-coating, constitutes a limiting step in their fabrication. The dipcoating method for dye loading requires the longest portion of fabrication time (an average of $16 \mathrm{~h})^{6}$ and has remained essentially unchanged since the modern DSSC was introduced by O'regan and Grätzel in 1991. ${ }^{7}$ The conventional dye-loading technique is not only inefficient, in terms of duration, but also has other drawbacks, including requiring organic solvents, ${ }^{\mathbf{8}, 9}$ generating significant dye waste, ${ }^{\mathbf{6}}$ and sensitization under dark conditions. ${ }^{\mathbf{1 0}}$ Moreover, dip-coating requires a high concentration of dye, which increases the probability of dye

${ }^{a}$ Department of Chemistry and Biochemistry, South Dakota State University, Brookings, SD 57007, USA. E-mail: brian.logue@sdstate.edu; Tel: +1-605-688-6698

${ }^{b}$ Department of Electrical Engineering and Computer Science, South Dakota State University, Brookings, SD 57007, USA aggregation, leading to degradation of cell performance., ${ }^{\mathbf{4 1 1 - 1 3}}$ To counteract dye aggregation in dip-coating, the dye solution is often prepared with co-adsorbents (e.g., cholic acid) ${ }^{\mathbf{1 4}}$ further complicating the fabrication process. ${ }^{15}$ Furthermore, after multiple uses of a single dye solution for dip-coating, the dye solution concentration changes and consistent sensitization requires cautious evaluation.

Functionalized Carboxylate Deposition (FCD), a vapor phase deposition technique, was introduced as an alternative sensitization technique in $2015 .^{6}$ FCD significantly reduces dye loading time, allows more efficient use of dye material, and avoids the use of solvents. Fig. 1 shows the comparison of FCD with conventional dip-coating dye loading. FCD utilizes dyes containing an electron withdrawing group in the $\alpha$-position to a carboxyl group. When these dyes are evaporated/ sublimated, they react chemically with hydroxyl groups which dominate the surface chemistry of $\mathrm{TiO}_{2}$ particles. ${ }^{16}$ As shown in Fig. 2, the FCD dyes covalently bond to a $\mathrm{TiO}_{2}$ surface hydroxyl with the carboxyl group of the dye through an esterification-type reaction. For FCD sensitization, dyes need to evaporate before degrading at elevated temperatures. ${ }^{6}$ However, at atmospheric pressure, many organic dyes degrade before sublimating/evaporating. Thus, a moderate to high vacuum is necessary to reduce the evaporation temperature. Mallam et al. demonstrated vapor phase deposition of two organic dyes, (Z)-2-cyano-3-(4-(dimethyl-amino)phenyl)acrylic acid and (Z)-2-cyano-3-(4-(diphenylamino)phenyl)-acrylic acid. ${ }^{6}$ They achieved comparable PCEs $(3.17 \%$ and $3.30 \%$, 


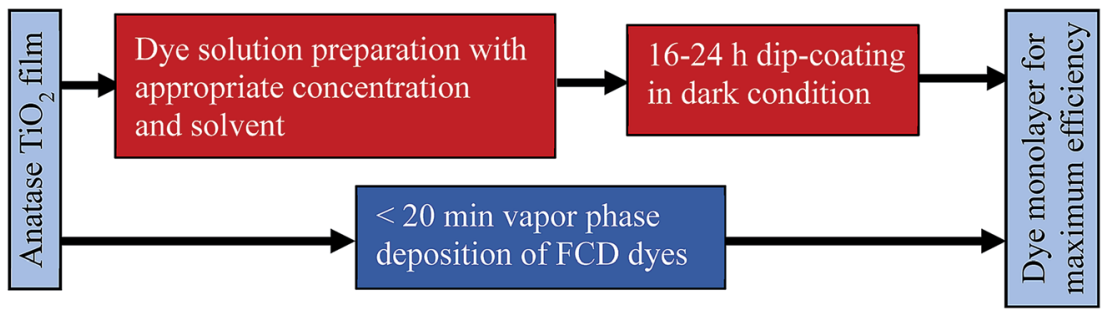

Fig. 1 Comparison of $\mathrm{FCD}^{6}$ and conventional dip-coating dye loading processes.

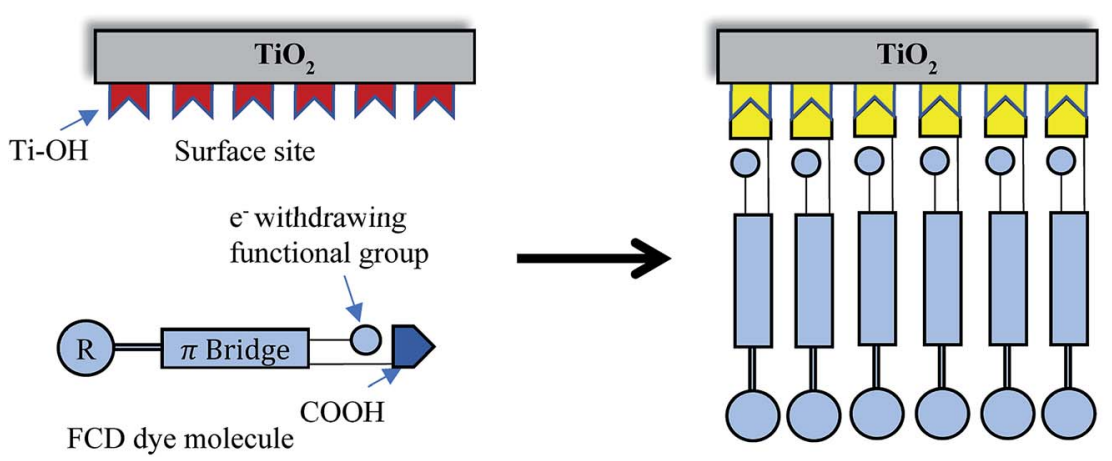

Fig. 2 An FCD dye molecule attaches through a $-\mathrm{COOH}$ group with surface $-\mathrm{OH}$ site.

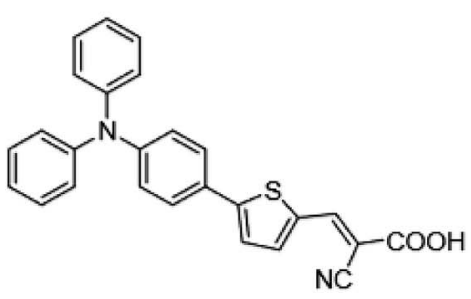

L1 dye

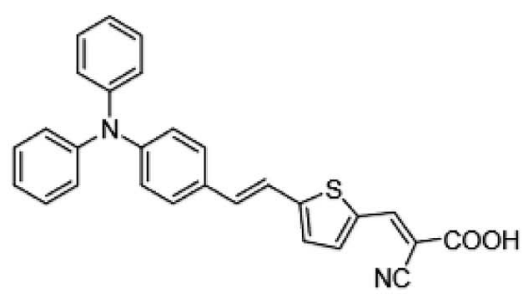

L2 dye

Fig. 3 Structures of the triphenylamine-based organic dyes used in this study. L1: 5-[4-(Diphenylamino)phenyl]thiophene-2-cyanoacrylic acid and L2: 3-(5-(4-(diphenylamino)styryl)thiophen-2-yl)-2-cyanoacrylic acid.

respectively) with that of dip-coating $(2.62 \%$ and $3.37 \%$, respectively), but the two dyes used were themselves relatively inefficient.

In order for FCD to be more broadly accepted as an alternative sensitization technique to dip-coating, there is a need to demonstrate broader applicability, especially for larger dyes. Specifically, because of instrument limitations, Mallam et al. used a relatively high pressure of 500 mTorr to sublimate two dyes $\left(\mathrm{MW}<340 \mathrm{~g} \mathrm{~mol}^{-1}\right)$ at 180 and $210^{\circ} \mathrm{C}$, respectively. Here, we report the rapid (<20 min) sensitization of photoanodes with two triphenylamine-based organic dyes, L1 (5-[4(diphenylamino)phenyl]thiophene-2-cyanoacrylic acid; MW = $422.50 \mathrm{~g} \mathrm{~mol}^{-1}$ ), and L2 (3-(5-(4-(diphenylamino)styryl) thiophen-2-yl)-2-cyanoacrylic acid; $\left.\mathrm{MW}=448.54 \mathrm{~g} \mathrm{~mol}^{-1}\right)$ (Fig. 3), and compare the power conversion efficiencies (PCEs) of DSSCs produced via FCD and the conventional dip-coating method.

\section{Experimental procedures}

\subsection{Materials}

Fluorine doped tin oxide (FTO) glass substrates were purchased from Hartford Glass Co., Indiana, USA. Nanocrystalline $\mathrm{TiO}_{2}$ (Ti-Nanoxide T/SP), scattering $\mathrm{TiO}_{2}$ (Ti-Nanoxide R/SP), $\mathrm{I}_{3}{ }^{-} / \mathrm{I}^{-}$ electrolyte (Iodolyte HI-30), activated platinum solution (Platisol T), and thermoplastic sealant (Meltonix 1170-60) were purchased from Solaronix (Aubonne, Switzerland). Organic dyes, L1 (5-[4-(diphenylamino)phenyl]thiophene-2-cyanoacrylic acid) and L2 (3-(5-(4-(diphenylamino)styryl)thiophen-2-yl)-2cyanoacrylic acid), were acquired from Dyenamo, Stockholm, Sweden. All materials were used as received.

\subsection{Photoanode preparation}

FTO substrates were cleaned by sonication in aqueous solutions of dodecyl sulfate sodium salt, deionized (DI) water, 
acetone, and 2-propanol each for $15 \mathrm{~min}$, followed by UV exposure for $15 \mathrm{~min}$. A layer of nanocrystalline $\mathrm{TiO}_{2}$ (TiNanoxide T/SP, Solaronix) of $0.25 \mathrm{~cm}^{2}$ was deposited via doctor blading and then sintered at $450{ }^{\circ} \mathrm{C}$ for $\sim 30 \mathrm{~min}$. A second nanocrystalline $\mathrm{TiO}_{2}$ layer was deposited according to the same procedure to achieve a thickness of $\sim 10 \mu \mathrm{m}$. $\mathrm{A} \mathrm{TiO}_{2}$ light scattering layer of Ti-Nanoxide R/SP (particle size $>100$ $\mathrm{nm}$ ) was then screen printed on top of the mesoporous layer and sintered at $450{ }^{\circ} \mathrm{C}$ for $30 \mathrm{~min}$. The photoanode was then treated by immersing in a $0.1 \mathrm{M}$ solution of $\mathrm{HCl}$ for $2 \mathrm{~h}$. Then the photoanode was dried with compressed $\mathrm{N}_{2}$ gas.

\subsection{Functionalized carboxylate deposition}

A customized FCD instrument (schematic shown in Fig. 4) consisting of a turbo pump, rotary vane pump, mass flow controller, and custom vacuum chamber was used to create 0.1 mTorr vacuum inside a $1.6 \mathrm{~L}$ vacuum chamber. Approximately $100 \mu \mathrm{g}$ of FCD dye was placed into a $1.9 \mathrm{~mL}$ vial. The photoanode was placed on top of the glass vial with the $\mathrm{nc}^{-\mathrm{TiO}_{2}}$ in the center of the vial opening, facing into the vial. A metal jacket was placed around the vial and the vial was placed on top of an electric hot plate. With a lab-jack, the hotplate raised up to the vacuum chamber, and a vacuum was applied via rotary vane and turbo pumps. When a vacuum of $\sim 3$ mTorr was reached, the hotplate was heated to $\sim 260{ }^{\circ} \mathrm{C}$ for the $\mathrm{L} 1$ dye and $\sim 280{ }^{\circ} \mathrm{C}$ for the $\mathrm{L} 2$ dye and held at that temperature for the desired amount of time. Following evaporation of the dye, the heater was turned off, and the chamber was cooled, unassisted, to $150{ }^{\circ} \mathrm{C}$. The vacuum chamber was then filled with argon gas until atmospheric pressure was reached. The sensitized photoanodes were then rinsed sequentially with anhydrous ethanol and acetone to remove excess dye. The photoanodes were then dried with compressed $\mathrm{N}_{2}$ gas. Excess dye in the wash solution was recovered by allowing the ethanol and acetone to evaporate unassisted in a hood.

After the efficiency was measured, the DSSCs were deconstructed and the photoanodes were rinsed with acetonitrile to remove the residual electrolyte and then dried with compressed
$\mathrm{N}_{2}$ gas. The dye attached with the photoanodes was extracted by dipping them in $3 \mathrm{~mL}$ of a $0.1 \mathrm{M} \mathrm{NaOH}$ solution for $24 \mathrm{~h}$.

\subsection{Dip-coating}

For dip-coating, dye solutions $(0.3 \mathrm{mM})$ were prepared in anhydrous ethanol. After the dye solution was stirred for $\sim 1 \mathrm{~h}$, it was filtered through a $0.2 \mu \mathrm{m}$ syringe filter (with polytetrafluoroethane membrane). Prepared photoanodes were soaked in the dye solutions for $\sim 24 \mathrm{~h}$ under dark conditions to attach dye molecules to the porous- $\mathrm{TiO}_{2}$ surface. The dye-sensitized photoanodes were rinsed with anhydrous ethanol to remove excess dye and then dried under nitrogen.

\subsection{DSSC fabrication}

Activated platinum solution (i.e., Platisol T) was spin coated at $2000 \mathrm{rpm}$ for $10 \mathrm{~s}$ on top of the FTO substrates followed by sintering at $450{ }^{\circ} \mathrm{C}$ for $15 \mathrm{~min}$ to prepare the counter electrode (CE). The CE was then cooled, unassisted, for $\sim 40$ min until it reached room temperature. The $\mathrm{CE}$ was assembled with the photoanode using the thermoplastic sealant. A channel of approximately $1.5 \mathrm{~mm}$ width (through the entire cell) was kept for electrolyte injection. The $\mathrm{I}_{3}{ }^{-} / \mathrm{I}^{-}$electrolyte solution was then injected through the reserved channel in between the photoanode and CE. The channel openings were glued using a conventional hot glue gun.

\subsection{Characterization and evaluation}

Current density-voltage $(J-V)$ characteristics of DSSCs were evaluated under an AM1.5 illumination at a light intensity of $100 \mathrm{~mW} \mathrm{~cm}^{-2}$. A Xenon 40 lamp (Newport 67005) with an AM1.5 filter was used as the light source. The incident photon-tocurrent efficiency (IPCE) was measured as a function of wavelength from 350 to $800 \mathrm{~nm}$ using a xenon lamp connected to a Newport monochromator. The monochromatic light was focused onto the active area of the DSSCs. The IPCE data were calibrated with a National Renewable Energy Laboratory (NREL) calibrated reference cell. UV-Vis absorption spectra were acquired using an Agilent 8453 UV-Vis spectrophotometer.

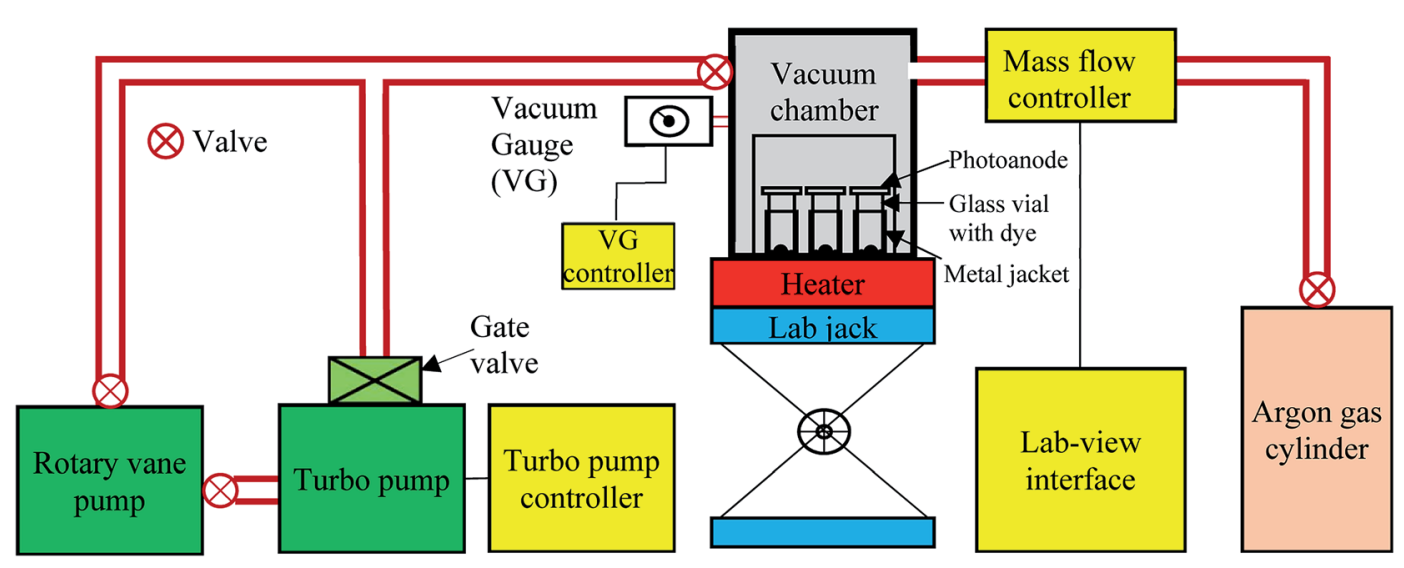

Fig. 4 Simplified schematic diagram of the FCD instrument. 


\section{Results and discussion}

\subsection{Photovoltaic performance}

Because of its nanoporous nature, an adequately sintered mesoporous $\mathrm{TiO}_{2}$ layer offers a large specific surface area ( $\sim 1000$ times the area of projected planar surface) ${ }^{17,18}$ For dipcoating, the solvent carries dye molecules through the nanopores of the nc- $\mathrm{TiO}_{2}$ film, in order to allow covalent bonding of the dye to the $\mathrm{TiO}_{2} \cdot{ }^{19}$ Because solution diffusion through nanopores is relatively slow, a long period of time (16-24 h) is necessary to fully sensitize the $\mathrm{nc}^{-\mathrm{TiO}_{2}}$ via dip-coating. Conversely, in the vapor phase, the dye molecules can freely diffuse into the $\mathrm{TiO}_{2}$ nanopores to quickly produce maximum surface coverage, as described by Mallam et al. ${ }^{6}$ Moreover, to produce a conformal coating, dye molecules must reach to the end of the pores of the nc-TiO ${ }_{2}$ layer, for which the solvent molecules, used for dip-coating, can actually act as a barrier, preventing full dye coverage. In FCD, the gaseous dye molecules have comparatively easy access through the nanopores.

Fig. 5 shows the current density-voltage $(J-V)$ characteristics curves of L1 and L2 dye-based DSSCs fabricated with dipcoating (for $\sim 24 \mathrm{~h}$ ) and FCD (at different durations). Table 1 summarizes the photovoltaic performances of these DSSCs. For both L1 and L2 dyes, the short circuit current density $\left(J_{\mathrm{sc}}\right)$ increases with the FCD duration up to an optimum duration. Though the molecular size of L2 is slightly larger than the L1, surprisingly, the optimum duration for L2 dye was found at a shorter deposition period (12 min) than that of L1 dye (16 min). This reveals that the specific dye structure, leading to different surface orientation, may affect the surface bonding kinetics. ${ }^{4,20-22}$

For prolonged FCD periods (i.e., $20 \mathrm{~min}$ for $\mathrm{L} 1$, and $16 \mathrm{~min}$ for L2), the $J_{\mathrm{sc}}$ values decreased, leading to decreased cell efficiencies. The lower $J_{\mathrm{sc}}$ values, at longer deposition times, were likely caused by dye aggregation. Aggregation leads to nonsurface bound dye molecules, resulting in photon capture without injection of the excited electron into the conduction band of $\mathrm{TiO}_{2}$, causing nonradiative decay of the electron from excited state to the ground state. ${ }^{\mathbf{4 , 6}}$

Although the $J_{\mathrm{sc}}$ values increased markedly with deposition time until the optimum FCD duration was reached, the $V_{\mathrm{oc}} \mathrm{S}$ (for both L1 and L2 dyes) were little affected (with $\sim 0.65 \mathrm{~V}$ for L1, $\sim 0.63 \mathrm{~V}$ for L2). This was expected since DSSC photovoltage is determined by the difference between the conduction-band
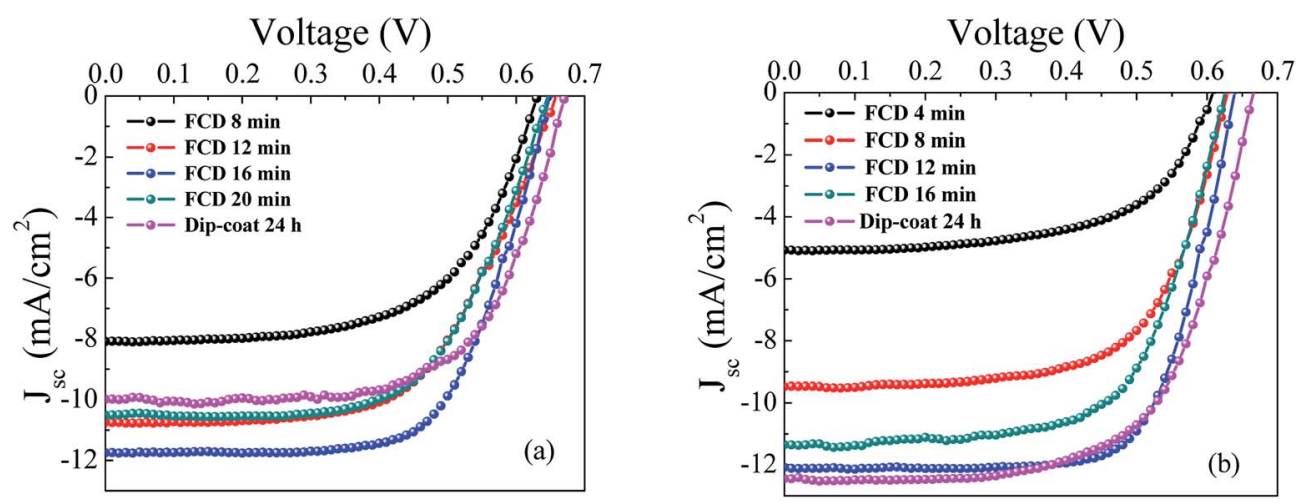

Fig. 5 Comparison of photovoltaic performance for dip-coating and FCD-based DSSCs (a) J-V curves of L1-based DSSCs; an optimum 16 min FCD shows significantly better performance compared to $24 \mathrm{~h}$ dip-coating (b) $\mathrm{J}-V$ curves of L2-based DSSCs; an optimum 12 min FCD shows equivalent performance to $24 \mathrm{~h}$ dip-coating.

Table 1 Current density-voltage $(\mathrm{J}-\mathrm{V}$ ) and IPCE characteristics of $\mathrm{L} 1$ and $\mathrm{L} 2$-dye based DSSCs fabricated with FCD and dip-Coating, and UV-Vis absorbance of dyes desorbed from the photoanodes

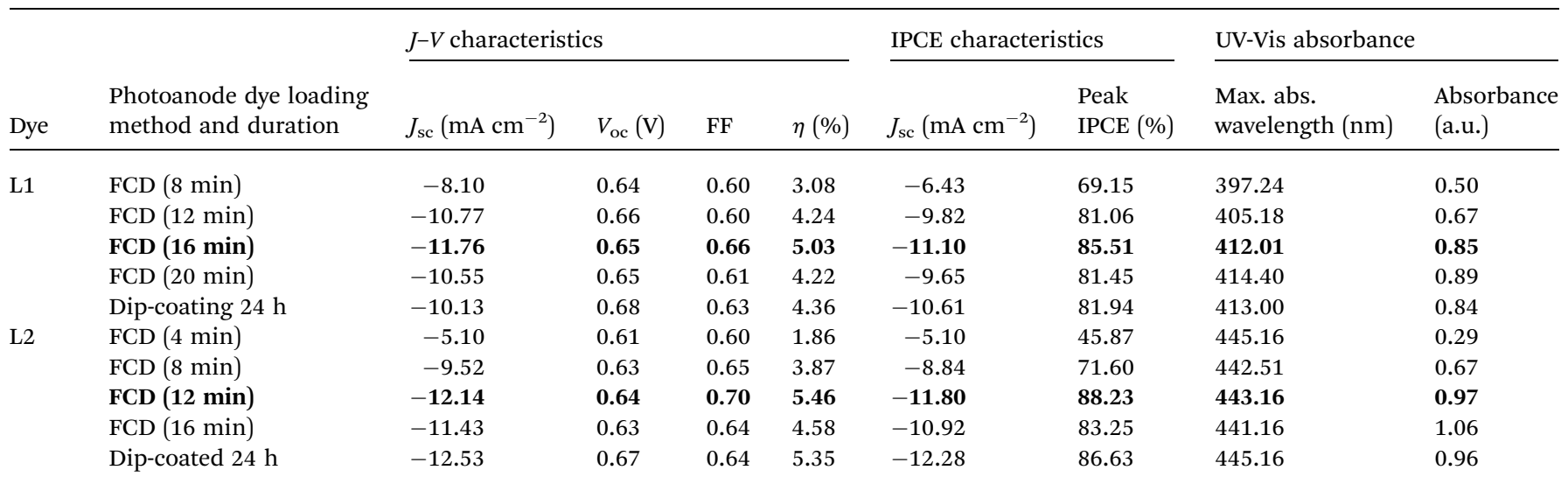


edge of $\mathrm{TiO}_{2}$ and the redox potential of the electrolyte, ${ }^{23}$ and generally not dependent on dye coverage. However, there is a small difference in $V_{\mathrm{oc}}$ between FCD and dip-coated DSSCs for both $\mathrm{L} 1$ and $\mathrm{L} 2$ dyes. In both cases, dip-coated $V_{\mathrm{oc}} \mathrm{S}$ were slightly higher than those of FCD. The origin of this difference is not fully understood. However, though the $V_{\mathrm{oc}}$ for dip-coated DSSCs were slightly higher compared to the FCD DSSCs, the PCEs (of dip-coated DSSCs) were lower, especially for L1-dye based DSSCs. The PCE for dip-coated L1 DSSC was significantly lower than that of the FCD (4.36\% compared to $5.03 \%)$, mainly due to the lower $J_{\mathrm{sc}}\left(10.13 \mathrm{~mA} \mathrm{~cm}{ }^{-2}\right.$ compared to 11.76 $\mathrm{mA} \mathrm{cm}{ }^{-2}$ ). The low $J_{\mathrm{sc}}$ from dip-coating is likely due to nonconformal dye coating and/or inadequate sensitization. The maximum PCE and $J_{\mathrm{sc}}\left(5.2 \%\right.$, and $12.8 \mathrm{~mA} \mathrm{~cm} \mathrm{~cm}^{-2}$ respectively) for L1-based dip-coated DSSCs was reported by Liu et al. ${ }^{23}$ However, they used tert-butanol-acetonitrile $(1: 1)$ as a dye solvent, and $1 \mathrm{mM}$ deoxycholic acid (DCA) as a co-adsorbent. In our study, we used anhydrous ethanol as a solvent and no coadsorbent, which might limit solubility and increase aggregation, respectively, of the L1 dye. For L2-based DSSCs, the efficiency attained by dip-coating $(5.35 \%)$ was similar to that of FCD (5.46\%). These efficiencies are comparable with the world record efficiency $(5.94 \%){ }^{4}$ This study suggests that the dipcoating efficiency of structurally similar dyes (i.e., triphenylamine in this case) may be vastly different, while FCD (allowing free diffusion of dye vapor) is free from this problem.

\subsection{Incident photon-to-current conversion efficiency (IPCE)}

To compare the incident monochromatic photon-to-current conversion efficiency (IPCE) between FCD and dip-coated DSSCs, we measured the IPCE spectra (Fig. 6) as a function of wavelength. The maximum IPCEs for L1 and L2-based DSSCs (for both FCD and dip-coating) were around 420 and $440 \mathrm{~nm}$, respectively, which is consistent with Hagberg et al. ${ }^{24}$ However, there is a slight red shift in the peak IPCE for L2-based DSSC, when FCD was implemented for $4 \mathrm{~min}$. The $J_{\mathrm{sc}}$ values were calculated from IPCE spectra using eqn $(1)^{25}$ to compare with the $J_{\text {sc }}$ values obtained from the $J-V$ characteristics (Table 1).

$$
J_{\mathrm{sc}}=\int_{\lambda_{2}}^{\lambda_{1}} e \eta_{\mathrm{IPCE}}(\lambda) N_{\mathrm{Ph}}(\lambda) d \lambda,
$$

where $\eta_{\mathrm{IPCE}}(\lambda)$ and $N_{\mathrm{Ph}}(\lambda)$ are the IPCE and photon flux at wavelength, $\lambda$, respectively.

The calculated $J_{\text {sc }}$ values, shown in Table 1 , are consistent with those measured from $J-V$ curves.

\subsection{Dye adsorption}

To study dye attachment at different durations, dye was desorbed from the photoanodes and UV-Vis absorbance of the resulting solution was measured. Table 1 shows that the absorption peak intensity increases with FCD duration, resulting from increased dye loading with increased deposition duration. It is noticeable that a similar amount of dye was desorbed from the dip-coated $(\sim 24 \mathrm{~h})$ cells and the optimized FCD (16 min for L1 and 12 min for L2) cells for both dyes. For the L1-based DSSC, though equal amount of dye was attached for $16 \mathrm{~min}$ FCD and $\sim 24 \mathrm{~h}$ dip-coating, there is a marked difference in the PCE, which may be due to non-conformal dipcoating sensitization. After optimum FCD duration, both the PCE and the IPCE decreased even though the amount of dye extracted increased (Table 1). This decrement is likely due to the dye aggregation, which results in decreased $J_{\mathrm{sc}} \mathrm{s}$. This result is in agreement with the previous FCD study. ${ }^{6}$ There was a slight blue shift in absorbance spectra for less-concentrated L1 dye solutions (i.e., solutions correspond to 8 and $12 \mathrm{~min}$ FCD for L1based photoanodes). A concentration-dependent shift in absorbance spectra was also observed by Kitamura et al. for organic dyes. ${ }^{26}$ However, there is no shift in absorbance spectra for L2 dye.

\subsection{Reduction of dye waste}

Besides offering reduced deposition time, one of the major advantages of FCD is efficient use of DSSC dyes, which can be very costly. Dyes are more efficiently used for FCD in a number of ways. First, when dissolving a dye for dip-coating, even after stirring 1-2 hours, it is necessary to filter the solution to
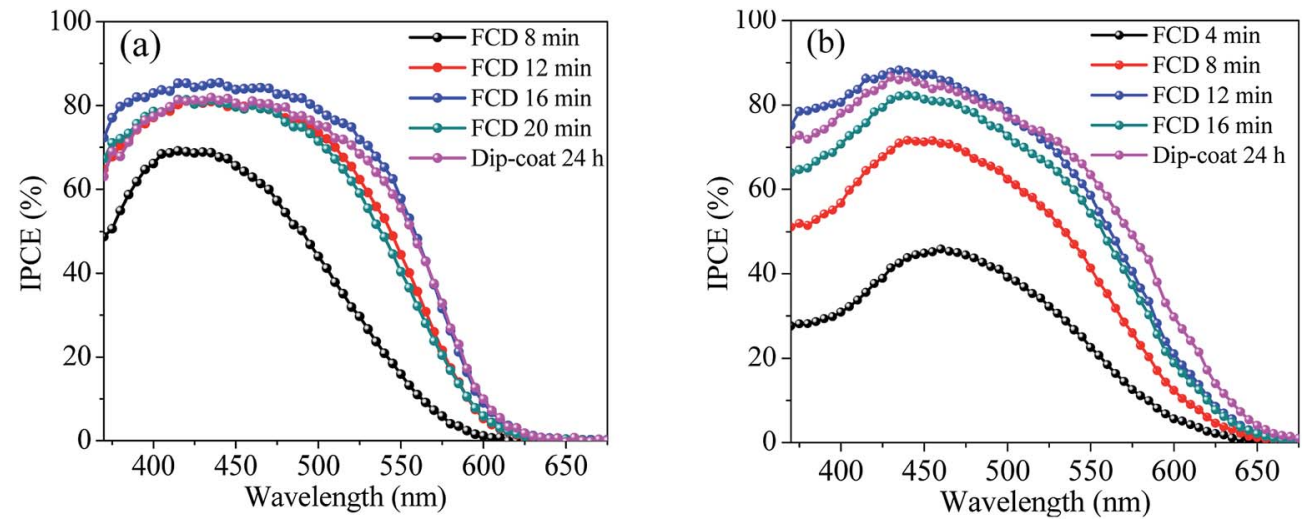

Fig. 6 Comparison of incident photon-to-current conversion efficiency (IPCE) for dip-coating and FCD-based DSSCS (a) IPCE spectra of L1 dye based DSSCs; peak IPCE increased with increased FCD duration until reached an optimum duration of 16 min (b) IPCE spectra of L2 dye based DSSCs; peak IPCE increased with increased FCD duration until reached an optimum duration of 12 min. 
remove the undissolved coarse dye grains to ensure they don't interfere with deposition. On the contrary, FCD doesn't require dissolution or filtering of the dye, reducing effort and dye waste.

Second, dye solutions for dip-coating are typically used multiple times, with storage in between. Storing dye-solutions for longer periods (e.g., >1 week) may easily lead to dye degradation (e.g., oxidation). Using this solution multiple times can cause poor sensitization and results in lower PCEs. FCD eliminates this issue by using a small amount of solid dye.

Third, during dip-coating, dye solutions must be kept in the dark (as the dye molecules are very light sensitive in solution) for long durations (i.e., 16-24 h). Whereas, in FCD, the vacuum chamber limits photoexcitation of the dye and the dye loading time is very small ( $\sim 20 \mathrm{~min})$, so the consequences of the light sensitivity are mitigated.

Dye use was further reduced in this study compared to that of Mallam et al. ${ }^{6}$ For the Mallam et al. study, a relatively large amount of dye was sublimated to sensitize multiple electrodes placed at different positions within the FCD chamber, leading to less-controlled and less-reproducible sensitization. For the current study, the FCD process was modified using a single vial for each photoelectrode (Fig. 4). The metal jacket ensured the dye did not deposit on the sides of the vial within the region of the jacket. At selected temperatures and pressures, only a small amount of dye was sublimated. In addition to photoelectrode surface, a small amount of dye deposited on the sides of the vials (above the metal jacket) and on the sides of the substrate. In Mallam et al. ${ }^{6}$ study, much more dye deposited on the walls of the FCD chamber. Following FCD, vials and the substrates were rinsed sequentially with anhydrous ethanol and acetone to remove excess dye. The excess dye in the wash solution was recovered, for future use, by evaporating the ethanol and acetone to dryness.

Based on all the advantages of FCD over dip-coating, and modification of the FCD process in this study, it was estimated that approximately, $20 \%$ of the dye is necessary for FCD as compared to dip-coating.

\section{Conclusion}

Although DSSC fabrication has undergone multiple modifications, the standard dye-loading technique (i.e., dip-coating) remains a major limiting issue taking more than $80 \%$ of the fabrication time. This study demonstrates that FCD with a higher vacuum ( $\sim 0.1$ mTorr) can produce DSSCs with equivalent or higher PCEs compared to dip-coating for larger molecular dyes (e.g., MW $>420 \mathrm{~g} \mathrm{~mol}^{-1}$ ) within a fraction of sensitization duration $(\sim 2 \%)$ of dip-coating. The shorter FCD dye-sensitization time (i.e., <20 min) was attributed to gaseous dye molecules' relatively easy diffusion capability through $\mathrm{TiO}_{2}$ nanopores to quickly produce maximum surface monolayer coverage. Besides the reduction of dye-loading duration, FCD demonstrated more efficient use of dye material and significant reduction of solvent use.

\section{Conflicts of interest}

There are no conflicts to declare.

\section{Acknowledgements}

We gratefully acknowledge funding support from the State of South Dakota Board of Regents through their Collaboration Grant 2016 program and the Surface Engineering Research Center. The views of the authors do not necessarily reflect the opinions of the South Dakota Board of Regents or the state of South Dakota.

\section{References}

$1 \mathrm{~S}$. Mathew, et al., Dye-sensitized solar cells with $13 \%$ efficiency achieved through the molecular engineering of porphyrin sensitizers, Nat. Chem., 2014, 6, 242.

2 M. K. Nazeeruddin, et al., Combined experimental and DFTTDDFT computational study of photoelectrochemical cell ruthenium sensitizers, J. Am. Chem. Soc., 2005, 127(48), 16835-16847.

3 C.-Y. Chen, et al., Highly efficient light-harvesting ruthenium sensitizer for thin-film dye-sensitized solar cells, ACS Nano, 2009, 3(10), 3103-3109.

4 A. Mishra, M. K. Fischer and P. Bäuerle, Metal-free organic dyes for dye-sensitized solar cells: from structure: property relationships to design rules, Angew. Chem., Int. Ed., 2009, 48(14), 2474-2499.

$5 \mathrm{~S}$. Ahmad, et al., Metal free sensitizer and catalyst for dye sensitized solar cells, Energy Environ. Sci., 2013, 6(12), 3439-3466.

6 V. Mallam, et al., Functionalized carboxylate deposition for rapid sensitization of dye-sensitized solar cells, Sol. Energy, 2016, 126, 128-136.

7 B. O'regan and M. Grätzel, A low-cost, high-efficiency solar cell based on dye-sensitized colloidal $\mathrm{TiO}_{2}$ films, nature, 1991, 353(6346), 737.

$8 \mathrm{H}$. Tian, et al., Effect of different dye baths and dye-structures on the performance of dye-sensitized solar cells based on triphenylamine dyes, J. Phys. Chem. C, 2008, 112(29), 11023-11033.

9 P. Shen, et al., Efficient triphenylamine dyes for solar cells: effects of alkyl-substituents and $\pi$-conjugated thiophene unit, Dyes Pigm., 2009, 83(2), 187-197.

$10 \mathrm{~T}$. Marinado, et al., How the nature of triphenylaminepolyene dyes in dye-sensitized solar cells affects the opencircuit voltage and electron lifetimes, Langmuir, 2009, 26(4), 2592-2598.

11 Z. Ning, Y. Fu and H. Tian, Improvement of dye-sensitized solar cells: what we know and what we need to know, Energy Environ. Sci., 2010, 3(9), 1170-1181.

12 D. P. Hagberg, et al., Molecular engineering of organic sensitizers for dye-sensitized solar cell applications, J. Am. Chem. Soc., 2008, 130(19), 6259-6266. 
13 N. Koumura, et al., Alkyl-Functionalized Organic Dyes for Efficient Molecular Photovoltaics, J. Am. Chem. Soc., 2006, 128(44), 14256-14257.

14 M. Rudolph, et al., Improvement of light harvesting by addition of a long-wavelength absorber in dye-sensitized solar cells based on $\mathrm{ZnO}$ and indoline dyes, J. Phys. Chem. $C, 2015,119(3), 1298-1311$.

15 G. B. Bodedla, et al., Benzimidazole-branched isomeric dyes: effect of molecular constitution on photophysical, electrochemical, and photovoltaic properties, J. Org. Chem., 2016, 81(2), 640-653.

16 S. M. George, B. Yoon and A. A. Dameron, Surface Chemistry for Molecular Layer Deposition of Organic and Hybrid Organic-Inorganic Polymers, Acc. Chem. Res., 2009, 42(4), 498-508.

17 C.-P. Lee, et al., Recent progress in organic sensitizers for dye-sensitized solar cells, RSC Adv., 2015, 5(30), 2381023825.

18 D. H. Kim, et al., Atomic Layer Deposition for Sensitized Solar Cells: Recent Progress and Prospects, Adv. Mater. Interfaces, 2016, 3(21), 1600354.

19 K.-J. Hwang, et al., Dye adsorption mechanisms in $\mathrm{TiO}_{2}$ films, and their effects on the photodynamic and photovoltaic properties in dye-sensitized solar cells, Phys. Chem. Chem. Phys., 2015, 17(34), 21974-21981.
$20 \mathrm{~J}$. Wiberg, et al., Distance and Driving Force Dependencies of Electron Injection and Recombination Dynamics in Organic Dye-Sensitized Solar Cells, J. Phys. Chem. B, 2010, 114(45), 14358-14363.

21 S. K. Eriksson, et al., Geometrical and energetical structural changes in organic dyes for dye-sensitized solar cells probed using photoelectron spectroscopy and DFT, Phys. Chem. Chem. Phys., 2016, 18(1), 252-260.

22 I. Obotowo, I. Obot and U. Ekpe, Organic sensitizers for dyesensitized solar cell (DSSC): properties from computation, progress and future perspectives, J. Mol. Struct., 2016, 1122, 80-87.

23 W.-H. Liu, et al., Simple organic molecules bearing a 3,4ethylenedioxythiophene linker for efficient dye-sensitized solar cells, Chem. Commun., 2008, (41), 5152-5154.

24 D. P. Hagberg, et al., Tuning the HOMO and LUMO energy levels of organic chromophores for dye sensitized solar cells, J. Org. Chem., 2007, 72(25), 9550-9556.

25 Y. Xie, et al., Electrolyte Effects on Electron Transport and Recombination at ZnO Nanorods for Dye-Sensitized Solar Cells, J. Phys. Chem. C, 2010, 114(41), 17880-17888.

26 T. Kitamura, et al., Phenyl-conjugated oligoene sensitizers for $\mathrm{TiO}_{2}$ solar cells, Chem. Mater., 2004, 16(9), 1806-1812. 